\title{
Check-list de Capparaceae do estado de Mato Grosso do Sul, Brasil
}

\author{
Thales Panfiglio', Xavier Cornejo² \& Maria Ana Farinaccio³
}

\begin{abstract}
'Universidade Federal de Mato Grosso do Sul, Centro de Ciências Biológicas e da Saúde,
Cidade Universitária, s/n. Caixa Postal 549, CEP 79070-900, Campo Grande, MS, Brasil. thales panfiglio@outlook.com ${ }^{2}$ Universidad de Guayaquil, Facultad de Ciencias Naturales, Herbario GUAY, P. O. Box. 09-01-10634, Guayaquil, Ecuador. 3Universidade Federal de Mato Grosso do Sul, Herbário COR, Campus Pantanal, Av. Rio Branco, 1270, 79304-902, Corumbá, MS, Brasil.
\end{abstract}

Recebido em 27.IX.2014.

Aceito em 3.VIII.2016

DOI 10.21826/2446-8231201873s174

RESUMO - Este check-list tem objetivo de atualizar as informações sobre as Capparaceae de Mato Grosso do Sul. Os resultados obtidos demonstram que o número total de táxons para o estado é de 12 espécies em seis gêneros e o número de espécie reconhecidas no Brasil passa para 29 em 12 gêneros. Novos registros de ocorrência para o país foram detectados, um gênero: Capparicordis Iltis \& Cornejo e duas espécies: Capparicordis tweediana (Eichler) Iltis \& Cornejo e Capparidastrum humile (Hassl.) Cornejo \& Iltis, além desses, também são reportados pela primeira vez em Mato Grosso do Sul: Capparidastrum frondosum (Jacq.) Cornejo \& Iltis, Cynophalla flexuosa (L.) J. Presl e Cynophalla mattogrossenssis (Pilg.) Cornejo \& Iltis.

Palavras-chave: Brassicales, Capparaceae, Pantanal

ABSTRACT - Checklist of Capparaceae of the state of the Mato Grosso do Sul, Brazil. This checklist updates the species information from the state of Mato Grosso do Sul. Our results demonstrate that the total number of taxa for the state is 12 species in six genera, whilst the number of species in Brazil is 29 in 12 genera. These include new records for Brazil, such as the genus: Capparicordis Iltis \& Cornejo and two species: Capparicordis tweediana (Eichler) Iltis \& Cornejo and Capparidastrum humile (Hassl.) Cornejo \& Iltis.. Capparidastrum frondosum (Jacq.) Cornejo \& Iltis, Cynophalla flexuosa (L.) J. Presl and Cynophalla mattogrossenssis (Pilg.) Cornejo \& Iltis, are also reported for first time in the state of Mato Grosso do Sul.

Keywords: Brassicales, Capparaceae, wamp

\section{INTRODUÇÃO}

Capparaceae Juss. e Brassicaceae Burnett são famílias proximamente relacionadas (Iltis 1957, Al-Shehbaz 1973, 1984, Dahlgren 1975, Takhtajan 1980, Cronquist 1981, Hauser \& Crovello 1982, Rollins 1993, Rodman et al. 1993, 1996, 1998), exceto por Hutchinson (1967). Estudos moleculares recentes confirmam esta proximidade entre Capparaceae e Brassicaceae (Hall et. al. 2002, 2004, Hall 2008, Iltis et. al. 2011). De acordo com APG (1998, 2003), Capparaceae e Brassicaceae estavam incluídas em Capparales, atualmente, ambas compõem a ordem Brassicales (APG 2009).

Em Capparaceae, já foram reconhecidas duas subfamílias, Cleomoideae e Capparoideae (APG 1998, 2003), no entanto, alguns autores percebiam que elas eram muito distintas (e.g., Shaw 1965, Hutchinson 1967), e consideravam a elevação do status para famílias. De acordo com Hall et. al. (2002, 2004, Hall 2008), com base nos seus estudos moleculares, a subfamília Cleomoideae emergiu como irmã das Brassicaceae, o que torna a família Capparaceae, assim circunscrita, um grupo parafilético.
Sendo assim, Hall et. al. (2002), defende a quebra de Brassicaceae s.1.: [Brassicaceae s.str., Capparaceae s.1. (com Cleomoideae incluídas)] (APG 1998, 2003) e o reconhecimento das três famílias monofiléticas: Capparaceae, Cleomaceae Horan. e Brassicaceae (APG 2009).

Deste modo, Capparaceae é aqui tratada como uma família distinta de Brassicaceae s.l., que emerge como grupo irmão de um clado que inclui Cleomaceae e Brassicaceae (Stevens 2001 onwards). Isso confere uma mudança do que conhecíamos anteriormente de gêneros e espécies pertencentes a cada uma dessas famílias.

A família apresenta distribuição pantropical, sendo mais evidente em habitats tropicais sazonalmente secos (Hall et. al. 2002). Apresenta 25 gêneros com 440 espécies distribuídas no Velho e Novo mundo. Dessas, 110 espécies em 20 gêneros são Neotropicais (Cornejo \& Iltis 2011, Cornejo \& Marques 2012). Até o momento, no Brasil são reconhecidos 11 gêneros e 27 espécies (Cornejo \& Marques 2012).

As Capparaceae são exclusivamente lenhosas e apresentam frutos carnosos sem replo, características que as diferenciam das Brassicaceae e Cleomaceae que são 
herbáceas e possuem frutos secos, com replo (Hall et. al. 2002, Hall 2008).

Capparaceae apresenta grande variação no hábito, fruto e características florais (Heywood 1993, Cornejo \& Iltis 2009). Pode incluir árvores, arbustos e raramente (no Novo Mundo) lianas, glabros ou pubescentes. As folhas são simples, alternas, usualmente em espiral ou dística, raramente opostas, frequentemente coriáceas, subséssil a peciolada; estípulas diminutas ou ausentes. Inclui tanto flores zigomorfas quanto actinomorfas e uma grande variação no número de estames pode ser observada 6 a 250 (Endress 1992, Iltis et. al. 2011).

\section{Principais Grupos de Pesquisa}

No Brasil não são conhecidos grupos de pesquisa relacionados à taxonomia e sistemática das Capparaceae, destacamos os trabalhos de Xavier Cornejo (GUAY/NY).

\section{Principais Lacunas do Conhecimento}

Como principais lacunas do conhecimento sobre a família Capparaceae no estado do Mato Grosso do Sul podemos citar: a) estudos de polinização e dispersão, já que os polinizadores, predadores dos frutos e dispersores das sementes da maioria das espécies desta família na região Neotropical são desconhecidos; b) estudos fenológicos, uma vez que se desconhecem os aspectos fenológicos das espécies. Portanto, é necessário levantar registros dos períodos de floração e frutificação das Capparaceae e c) estudos de horticultura, já que algumas espécies de Capparaceae poderiam ser cultivadas com fins ornamentais ou em reflorestamentos, porém, o ciclo biológico das espécies e seu manejo são desconhecidos.

\section{MATERIAL E MÉTODOS}

A elaboração desta lista partiu da publicação de Dubs (1998) que representa uma síntese para as Angiospermas do estado de Mato Grosso, quando este incluía em seus limites o estado de Mato Grosso do Sul. Os dados foram complementados com um levantamento no acervo do Herbário da Universidade Federal de Mato Grosso do Sul, campus Campo Grande (CGMS), sendo este o herbário base para a citação dos espécimes testemunhos ("vouchers"). Também foi consultado o banco de dados do projeto CRIA (2012). A partir daí foi feita uma comparação com a Lista de Espécies da Flora do Brasil 2012 (LEFB).

Os acervos encontrados com os materiais testemunhos ("vouchers") para representar as espécies de Capparaceae do estado de Mato Grosso do Sul foram: CGMS e NY, seguidos do LPB, MBM e UB. A abreviação dos nomes dos autores de táxons e citação das obras originais de publicações dos nomes científicos seguiu a proposta no site "Tropicos" (2012), do "Missouri Botanical Garden", mantendo, assim, um mesmo padrão.

Para a elaboração da lista dos táxons, foram adotados os conceitos mais recentes para os grupos aqui tratados (Hall et al. 2002, Hall 2008, Cornejo et. al. 2008, Iltis \&
Cornejo 2007, 2010a, b, Cornejo \& Iltis 2008a, b, c, 2010a, b, Iltis et al. 2011). Os acrônimos dos herbários estão em acordo com Thiers (2008).

\section{RESULTADOS E DISCUSSÃO}

Apresentamos a seguir a lista de espécies Capparaceae do estado de Mato Grosso do Sul, com seus respectivos basiônimos (quando se tratar de mudanças recentes) e do material examinado.

Anisocapparis Cornejo \& Iltis, J. Bot. Res. Inst. Texas 2(1): 62-67, f. 1, 2, 4 a,b. 2008.

Anisocapparis speciosa (Griseb.) Cornejo \& Iltis. Capparis speciosa Griseb.

Material examinado: F. Matos-Alves 601 (CGMS) Chaco e Pantanal: Corumbá e Porto Murtinho

Capparicordis Iltis \& Cornejo, Brittonia 59(3): 246-254, f. 1-4. 2007.

Capparis tweediana Eichler

Material examinado: D.R.C. Padilha 28 (CGMS).

Chaco: Corumbá e Porto Murtinho.

Capparidastrum (DC.) Hutch, Gen. Fl. Pl. 2: 310. 1967. Capparidastrum frondosum (Jacq.) Cornejo \& Iltis. Capparis frondosa Jacq.

Material examinado: R. Aguilar 6828 (NY). Pantanal: Corumbá.

Capparidastrum coimbranum (Cornejo \& Iltis) Cornejo \& Iltis. Capparis coimbrana Cornejo \& Iltis

Material examinado: $M$. Nee 38561 (LPB). Pantanal: Corumbá.

Capparidastrum humile (Hass1.) Cornejo \& Iltis. Capparis humilis Hassl.

Material examinado: V. Pott 1518 (MBM). Pantanal: Corumbá. Rara no estado.

Capparidastrum osmanthum (Diels) Cornejo \& Iltis. Capparis osmantha Diels

Material examinado: J.A. Ratter R.6114 (NY). Pantanal: Corumbá. Rara no estado.

Crateva L., Sp. P1. 1: 444. 1753.

Crateva tapia L.

Material examinado: I.M. Bortolotto 653 (CGMS). Chaco e Pantanal: Corumbá, Porto Murtinho e Miranda.

Cynophalla (DC.) J. Presl, Prir. Rostlin 2: 275.1825.

Cynophalla declinata (Vell.) Iltis \& Cornejo. Capparis declinata Vell.

Material examinado: J.A. Ratter R.5950 (NY). Corumbá: Pantanal.

Cynophalla flexuosa (L.) J. Presl. Capparis flexuosa L. 
Material examinado: J.E. Paula 1678 (UB). Sem localidade. Rara no estado.

Cynophalla mattogrossenssis (Pilg.) Cornejo \& Iltis. Capparis mattogrossensis Pilger

Material examinado: M. Rocha 38 (CGMS). Pantanal:

Corumbá. Rara no estado.

Cynophalla retusa (Griseb.) Cornejo \& Iltis.

Capparis retusa Griseb.

Material examinado: G.A. Damasceno-Junior 3152 (CGMS). Cerrado, Chaco, Pantanal: Corumbá, Porto Murtinho e Ladário. Frequente.

Monilicarpa Cornejo \& Iltis, J. Bot. Res. Inst. Texas 2(1): 67-73, f. 3, 5, 6. 2008.

Monilicarpa brasiliana (Banks ex DC.) Cornejo \& Iltis . Capparis brasiliana Banks ex DC

Material examinado: C. Kozera, 1651 (MBM). Cerrado: Eldorado.

Destacamos que as espécies, tradicionalmente, identificadas como Capparis L., foram removidas do gênero e realocadas em diferentes táxons conforme Hall (2008). Os resultados obtidos representam uma atualização do conhecimento sobre as Capparaceae do Brasil e de Mato Grosso do Sul. Durante a elaboração deste check-list, foram detectados novos registros para o Brasil: o gênero Capparicordis e as espécies Capparicordis tweediana e Capparidastrum frondosum. Além desses, novos registros para o estado: Capparidastrum humile, Cynophalla flexuosa e Cynophalla mattogrossensis. Assim, o número total de táxons para o estado, quando comparado aos dados apresentados pela LEFB (Cornejo \& Marques 2012), aumentou de sete espécies em cinco gêneros para 12 espécies em seis gêneros e o número de espécie reconhecidas no Brasil passa para 29 em 12 gêneros.

\section{AGRADECIMENTOS}

Agradecemos ao Herbário Virtual da Flora e Fungos (INCT) pelo trabalho de integração das informações dos acervos dos herbários do país e aos pesquisadores Ms. Vali Pott e Dr. Arnildo Pott pelo apoio prestado

\section{REFERÊNCIAS}

Angiosperm Phylogeny Group- APG 1998. An Ordinal Classification for the Families of Flowering Plants. Annals of the Missouri Botanical Garden 85:531-553.

Angiosperm Phylogeny Group - APG 2003. An update of the Angiosperm Phylogeny Group classification for the orders and families of flowering plants: APG II. Botanical Journal of the Linnean Society 141:399-436.

Angiosperm Phylogeny Group - APG 2009. An update of the Angiosperm Phylogeny Group classification for the orders and families of flowering plants: APG III. Botanical Journal of the Linnean Society 161:105-121.

Al-Shehbaz, I.A. 1973. The biosystematics of the genus Thelypodium (Cruciferae). Contributions from the Gray Herbarium 204:3-148. 1984. The tribes of Cruciferae (Brassicaceae) in the southeastern United States. Journal of the Arnold Arboretum 65:343-373.
Centro de Referência em InformaçãoAmbiental- CRIA. Species Link e do SinBiota, Fundação de Amparo a Pesquisa do Estado de São Paulo. Disponível em: http://www.splink.org.br/index. Acessado em 25.06.2012.

Cornejo, X., Iltis, H.H. \& TOMB, A.S. 2008. Anisocapparis y Monilicarpa: Dos nuevos géneros de Capparaceae de América del Sur. Journal of the Botanical Research Institute of Texas 2(1):61-74.

Cornejo, X. \& Iltis, H.H. 2008a. The Reinstatement of Capparidastrum (Capparaceae). Harvard Papers in Botany 13(2):229-236.

2008b. A Revision of the American Species of the Genus Crateva (Capparaceae). Harvard Papers in Botany 13(1):121-135.

.2008c. New Combinations in South American Capparaceae. Harvard Papers in Botany 13(1):117-120.

2009. Hispaniolanthus: A new genus of Capparaceae endemic to Hispaniola. Harvard Papers in Botany 14(1):9-14.

.. 2010a. Nota Científica / Short Communication: Lectotypification and a new combination in Cynophalla (Capparaceae). Rodriguésia 61(1):153-155. Disponível em: http://rodriguesia.jbrj.gov.br. Acessado em 03.08.2012.

2010b. Studies in Capparaceae XXVII: Six new taxa and a new combination in Quadrella. Journal of the Botanical Research Institute of Texas 4(1):75-91.

.2011. Two new genera and three new combinations in Neotropical Capparaceae. Harvard Papers in Botany 16(1):65-70.

Cornejo, X. \& Marques, J.S. 2012. Capparaceae. In Lista de Espécies da Flora do Brasil. Jardim Botânico do Rio de Janeiro. Disponível em: http://floradobrasil.jbrj.gov.br. Acessado em 24.06.2012.

Cronquist, A. 1981. An integrated system of classification of flowering plants. Columbia University Press, New York, New York, USA. 1262p.

Dahlgren, R. 1975. System of classification of the angiosperms to be used to demonstrate the distribution of characters. Botaniska Notiser 128:119147.

Dubs, B. 1998. Prodromus Florae Mattogrossensis. Series B. v. 3. In Capparaceae. The Botany of Mato Grosso. Küsnach, Beltrona-Verlag, 49-50.

Endress, P.K. 1992. Evolution and floral diversity: the phylogenetic surroundings of Arabidopsis and Antirrhinum. International Journal of Plant Sciences 153:106-122.

Hauser, L.A. \& Crovello, T.J. 1982. Numerical analysis of generic relationships in Thelypodieae (Brassicaceae). Systematic Botany 7:249-268.

Hall, J.C., Sytsma, K.J. \& Iltis, H.H. 2002. Phylogeny of Capparaceae and Brassicaceae based on chloroplast sequence data. American Journal of Botany 89(11):1826-1842.

Hall, J.C., Iltis, H.H. \& Sytsma, K.J. 2004. Molecular Phylogenetics of Core Brassicales, Placement of Orphan Genera Emblingia, Forchhammeria, Tirania, and Character Evolution. Systematic Botany 29(3): 654-669.

Hall, J.C. 2008. Systematics of Capparaceae and Cleomaceae: evaluation of the generic delimitations of Capparis and Cleome using plastid DNA sequence data. Botany 86:682-696.

Heywood, V.H. 1993. Flowering plants of the world. Oxford University Press, New York, New York, USA, p. 118-119.

Hutchinson, J. 1967. The genera of flowering plants (Angiospermae) Based Principally on the Genera Plantarum of G. Benthan and J. D. Hooker. Clarendon Press, Oxford, UK, p. 303-317.

Iltis, H.H. 1957. Studies in the Capparidaceae III. Evolution and phylogeny of the Western North American Cleomoideae. Annals of the Missouri Botanical Garden 44:77-119.

Iltis, H.H. \& Cornejo, X. 2007. Studies in the Capparaceae XXX: Capparicordis, a new genus from the Neotropics. Brittonia 59(3): 245-254.

.. 2010a. Studies in the Capparaceae XXIX: Synopsis of Quadrella, a Mesoamerican and West Indian genus. Journal of the Botanical Research Institute of Texas 4(1):117-132.

2010b. Studies in Capparaceae XXVIII: The Quadrella cynophallophora complex. Journal of the Botanical Research Institute of Texas 4:93-115.

Iltis, H.H., Hall, J.C., Cochrane, T.S. \& Sytsma, K.J. 2011. Studies in the Cleomaceae I. On the Separate Recognition of Capparaceae, Cleomaceae, and Brassicaceae. Annals of the Missouri Botanical Garden 98:28-36.

Lista de Espécies da Flora do Brasil (LEFB). 2012. Banco de dados. Disponível em: http://floradobrasil.jbrj.gov.br/2012. Acessado em 25.06.2012. 
Rodman, J.E., Karol, K.G., Price, R.A. \& Sytsma, K.J. 1996. Molecules, morphology, and Dahlgren's expanded order Capparales. Systematic Botany 21:289-307.

Rodman, J.E., Price, R.A., Karol, K., Conti, E., Sytsma, K.J. \& Palmer, J.D. 1993. Nucleotide sequences of the rbcL gene indicate monophyly of mustard oil plants. Annals of the Missouri Botanical Garden 80:686-699.

Rodman, J.E., Soltis, P.S., Soltis, D.E., Sytsma, K.J. \& Karol, K.G. 1998. Parallel evolution of glucosinolate biosynthesis inferred from congruent nuclear and plastid gene phylogenies. American Journal of Botany 85:997-1006.

Rollins, R.C. 1993. The Cruciferae of continental North America: systematics of the mustard family from the Arctic to Panama. Stanford University Press, Stanford, California, USA. 996 p.
Shaw, A.H.K. 1965. Diagnoses of new families, new names, etc., for the seventh edition of Willis's 'Dictionary'. Kew Bulletin 18:249-273.

Stevens, P.F. 2001 onwards. Angiosperm Phylogeny Website. Version 12, July 2012. Disponível em: http://www.mobot.org/MOBOT/research/ APweb/. Acessado em 31.07.2012.

Takhtajan, A.L. 1980. Outline of the classification of flowering plants. Botanical Review 46:225-359.

Thiers, B. 2008. (continuamente atualizado). Index Herbariorum: A global directory of public herbaria and associated staff. New York Botanical Garden's Virtual Herbarium. Disponível em: http://sweetgum.nybg.org/ ih. Acessado em 29.07.2012.

Tropicos. Missouri Botanic Garden 2012. Disponível em: http://www. tropicos.org. Acessado em 28.06.2012. 